Progress in Nuclear Science and Technology

Volume 6 (2019) pp. 13-17

\title{
ARTICLE
}

\section{Study on leakage test of iodine adsorber using cyclohexane}

\author{
Jie $\mathrm{Yu}^{\mathrm{a}^{*}}$, Jianxing $\mathrm{Du}^{\mathrm{b}}$, Longjiang Wang ${ }^{\mathrm{a}}$, Yongguo $\mathrm{Li}^{\mathrm{a}}$, Taifei Qiao ${ }^{\mathrm{a}}$ and Yuan Zhang ${ }^{\mathrm{a}}$ \\ ${ }^{a}$ China Institute for Radiation Protection, Taiyuan 030006, Shanxi, China; ${ }^{b}$ Daya Bay Nuclear Power Operations and Management \\ Co. ltd, Shenzhen518000, Guangdong, China
}

\begin{abstract}
A new method for performance test of iodine adsorber, which is called cyclohexane method, is developed. Leakage simulation experiments and stability experiments using cyclohexane and freon respectively were conducted and compared to study the feasibility of using cyclohexane to replace Freon for in situ performance tests of iodine adsorber. In addition, the sorption and desorption behaviors of cyclohexane in impregnated activated carbon of iodine adsorber were investigated to study their effects on in situ mechanical leakage tests. For this reason, the effects of different carrier gas temperature, relative humidity, flow rate, and cyclohexane concentration in air were carefully investigated. Experimental results show that cyclohexane can be retained about $500 \mathrm{~min}$ in activated carbon bed therefore it can be used to replace freon to avoid its damage to atmosphere and temperature needs to pay attention in in situ performance tests because of its significant affection.
\end{abstract}

Keywords: cyclohexane; leakage test; iodine adsorber

\section{Introduction}

The effectiveness of iodine adsorbers, which are designed to remove radioactive iodine and its compounds to protect people and environment in nuclear air treatment systems, is critical to the safe operation of nuclear facilities. To verify whether their function meets design requirements, it is necessary to perform an in-place leak test after an iodine adsorber is installed. The installed iodine adsorber is also regularly tested during operation period to make sure it is always effective to remove radioactive iodine [1].

Three methods at present are used to evaluate the reliability of an iodine adsorber, radioactive methyl iodide method [2,3], non-radioactive methyl iodide method [4,5], and freon method [6,7]. Radioactive methyl iodide and non-radioactive methyl iodide methods can be used to directly measure the efficiency of iodine adsorbers to remove methyl iodine. Freon method measures only mechanical leakages caused by some interstices and defects of nuclear air treatment systems that radioactive iodine can easily penetrate from the leakage points but not go through the activated carbon layer, and a "Standard Test Method for Nuclear-Grade Activated Carbon" per ASTM D3803 in laboratory is needed to be combined to ensure the whole performance of iodine adsorbers to remove radioactive iodine. That is to say, in order to ensure an iodine adsorber installed in a nuclear air treatment system meet design requirements,

*Corresponding author. Email: flyyujie@126.com an in-place freon test is needed to validate that the mechanical leakage of this system is less than $0.05 \%$ and a laboratory test to verify that the efficiency of removing radioactive methyl iodide with impregnated activated carbon, which is the same batch with those filled in this iodine adsorber, is higher than $97 \%$.

Freon method is generally used in all nuclear power plants in United State and some nuclear power plants in China and its utilization is gradually limited because of the damage to ozone layer of atmosphere by freon reagents, therefore, EDF (France Power Group) company began to use cyclohexane as challenge gas to test the mechanical leakages of iodine adsorbers in ventilation systems in 2003 [8]. In order to prompt its application of this cyclohexane method in China, the feasibility of using cyclohexane to detect leakage rate of iodide adsorbers is studied, especially the influence of various factors on desorption behaviour of cyclohexane in activated carbon was studied by simulating different experimental conditions to provide useful information for the application of cyclohexane method in nuclear industry.

\section{Experimental section}

\subsection{Experimental principle}

When volatile organic gas contacts with porous solids, the organic components of the gas stream will be adsorbed on the solid surface, due to the presence of unbalanced molecular attraction. Iodine adsorbent leak rate detection can be achieved due to this 
features.

As shown in Figure 1, if the iodine adsorber itself is intact or installed correctly, the test medium can be completely retained by the activated carbon when the upstream is filled with organic gas that can be retained by the activated carbon. so the adsorbate concentration of downstream is very low. If there is a bypass leakage point in the iodine adsorber system, part of the organic gas directly flows into the downstream from the bypass leakage point without passing through the activated carbon layer, resulting in the rapid increase of the downstream concentration. Therefore, it is possible to determine whether there is a mechanical leak by measuring the concentrations of specific organic compounds in the upstream and downstream gas streams of the iodine adsorbers. Due to the weak adsorption of organic substance and activated carbon, the adsorbed organic gas is continuously adsorbed and desorbed in the activated carbon layer. After a certain period of purging, the adsorbed matter gradually desorbs from the activated carbon layer with the gas flow, and organic matter concentration will increase in the downstream.

Thus, an organic reagent that meets the following criteria can be used as a test reagent for the mechanical leak rate of an iodine adsorber: 1. Retention time on activated carbon is long enough. 2 . There is no disruptive effect on activated carbon. 3. It can be effectively

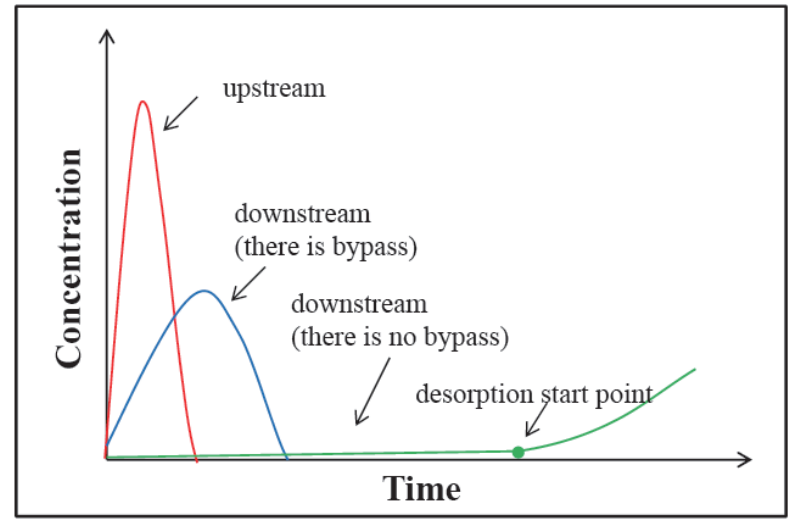

Figure 1. Schematic diagram of experimental principle. measured.

\subsection{Dynamic desorption test}

Activated carbon has different adsorption capacity on various conditions for the same organic matter, so the start time of the desorption is different. If the desorption starts too early, it will cause the mechanical leakage and desorption difficult to distinguish, resulting in the test invalid. Therefore, dynamic desorption test is carried out by simulating the possible flow conditions of NPP ventilation system to prove that the cyclohexane gas can be adsorbed by the activated carbon layer, and the desorption can not affect the judgment on the mechanical leakage rate at different humidity, temperature, flow rate and upstream challenge gas concentration.

An experimental device was established to study the dynamic desorption behavior of cyclohexane in an impregnated carbon bed (Figure 2). In order to prevent the condensed water from entering the activated carbon bed in air flow line, a gas-water separator is set at the outlet of the water bath humidifier. A buffer tank with temperature detector and humidity probe was set in front of the carbon bed to ensure the stability of the air flow, which can also display the real-time temperature and humidity of the airflow. When relative humidity and temperature of the airflow in pipeline can not meet the design requirements of experiment, a valve is opened to discharge the air flow out to avoid the unqualified airflow passing into the activated carbon test bed. A glass rotameter is used to control airflow not over its rated flow of $\pm 10 \%$. The activated carbon test bed includes 5 stainless steel cylinders of $25 \mathrm{~mm}$ diameter and $10 * 5 \mathrm{~mm}$ depth, filled with 8-16 mesh impregnated activated carbon of coconut shell. Experiments of dynamic desorption of cyclohexane under different conditions were carried out, concentrations of cyclohexane in airflow were analyzed by a PID (Photo Ionization Detector) gas chromatograph after sampled with a diaphragm pump sampler $[9,10]$.

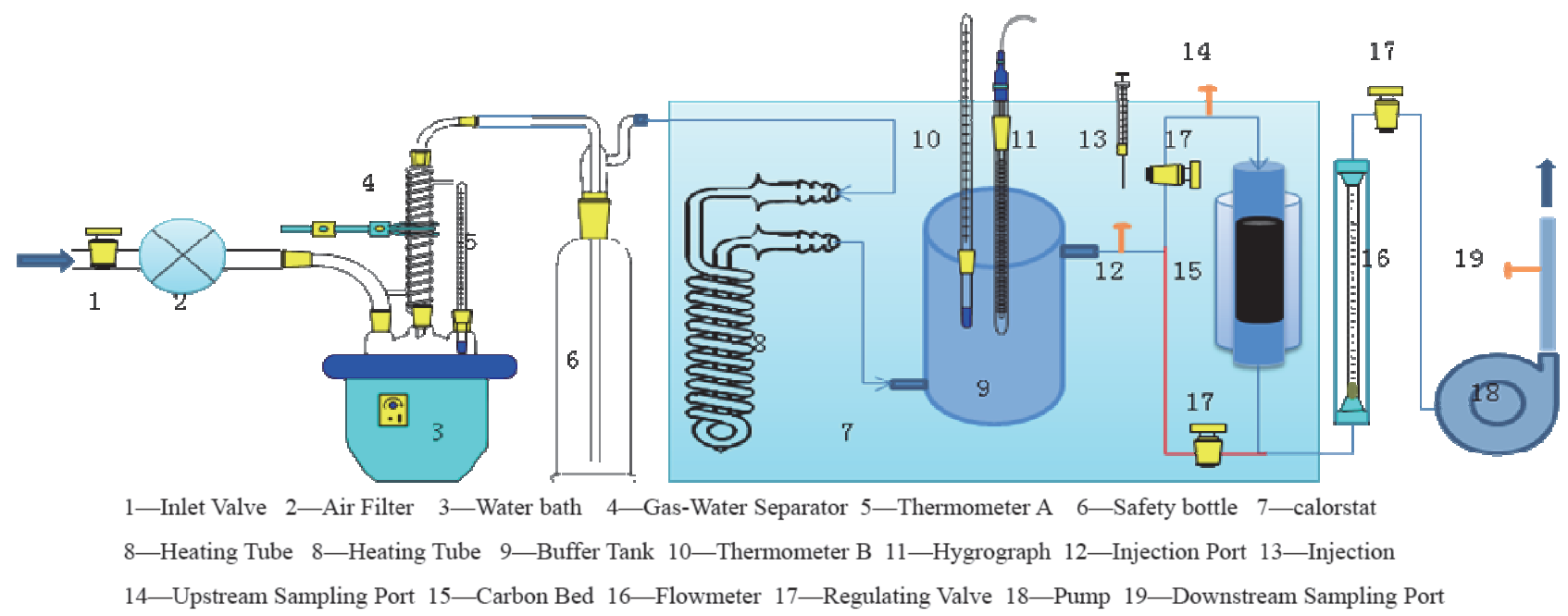

Figure 2. Schematic diagram of experimental device for cyclohexane desorption. 


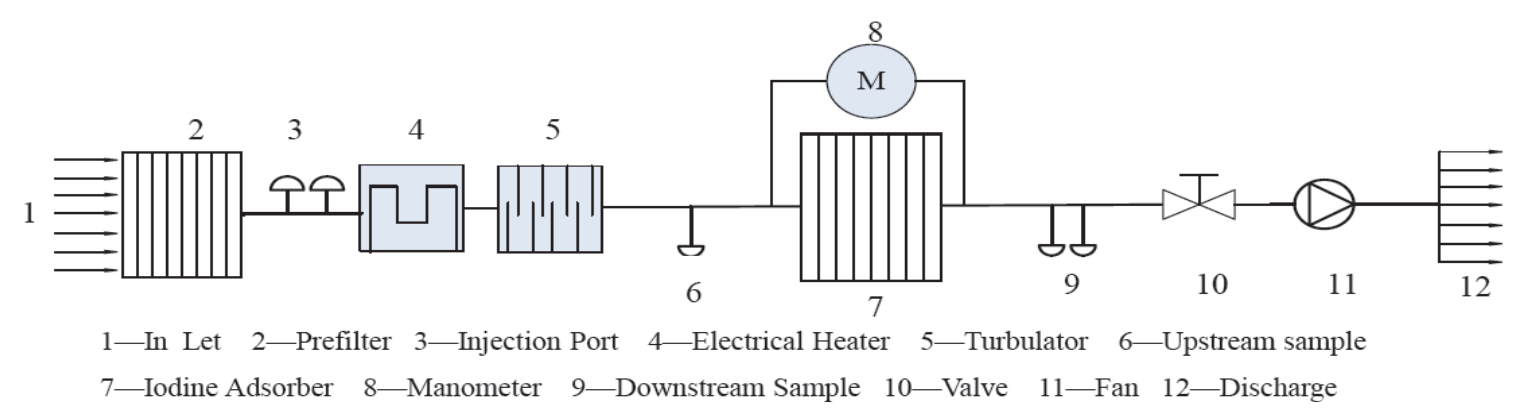

Figure 3. Flow diagram of experimental device for leakage rate test.

\subsection{Leakage test}

In order to verify the availability of cyclohexane method, a leakage rate test of iodine adsorber was conducted [8] using cyclohexane as test medium under the rated air flow. After the temperature and humidity of airflow met their preset values, a designed amount $(5 \mathrm{ml})$ of cyclohexane gas was injected into the upstream (1200 $\mathrm{m}^{3} / \mathrm{h}$ ) of the filter from the injection point in pulse mode. At the same time, started the sampling pump so that the upstream and downstream gas samples could be collected into Tedlar bags. The concentrations of the gas samples were measured by a gas chromatograph [9], the leakage rate was calculated with the following formula.

$$
\text { Leakage Rate } \mathrm{E}=\frac{\mathrm{C}_{\text {down }}}{C_{u p}}
$$

$C_{\text {down }}-$-Concentration of downstream sampling, ppm

$C_{u p}-$ Concentration of upstream sampling, ppm

\section{Results and discussion}

\subsection{Desorption test}

\subsubsection{Relative humidity}

The relationship between relative humidity and cyclohexane desorption is obtained in Figure 4. The result shows that the residence time is reducing with the increasing of relative humidity in airflow. This is mainly due to part of microporous blockage in activated carbon caused by water vapor in carrier gas and resulted decrease of adsorption surface area. The higher the

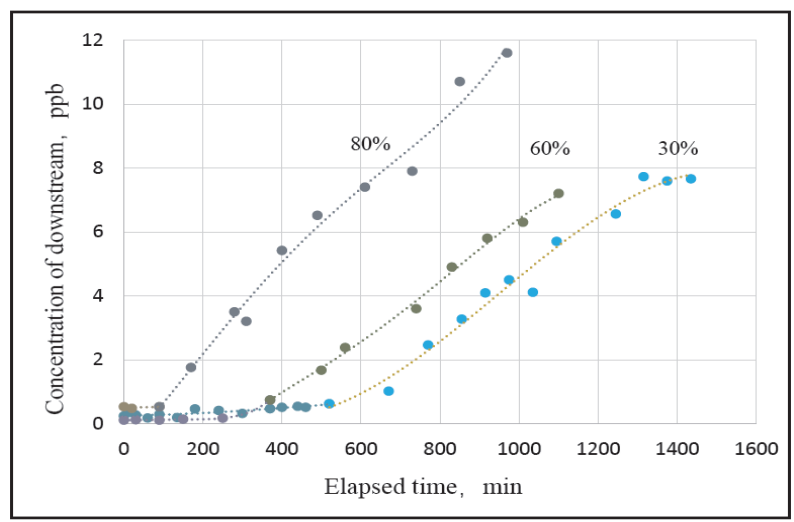

Figure 4. Desorption curve of cyclohexane with relative humidity relative humidity of carrier gas, the higher the degree of plugging and competing adsorption of water vapor in activated carbon, therefore the efficiency of cyclohexane adsorption is seriously degraded, and short desorption time as well as higher concentration of cyclohexane in downstream airflow behaved. When the relative humidity rises up to $80 \%$, there is still a long residence time. So, the leakage test can be performed at high relative humidity condition.

\subsubsection{Temperature}

Cyclohexane desorption curves of $40^{\circ} \mathrm{C}, 55^{\circ} \mathrm{C}$ and $70^{\circ} \mathrm{C}$ at ambient pressure and $30 \%$ relative humidity were displayed in Figure 5. The experimental result shows that. When the temperature of airflow is about $40^{\circ} \mathrm{C}$, the cyclohexane can be retained about $500 \mathrm{~min}$ on the activated carbon layer. When the temperature rises to $55^{\circ} \mathrm{C}$, the time of the cyclohexane concentration in downstream of air flow increases from 160 minutes after test commencement. And the temperature rises to $70^{\circ} \mathrm{C}$, the residence time shorts to $10 \mathrm{~min}$. This is mainly attributed to that the adsorption of cyclohexane on activated carbon is an exothermic process and therefore elevated temperature is conducive to cyclohexane desorption. Although the adsorption and desorption rate both increase with the rise of temperature, the desorption rate increases more rapidly than adsorption rate, thus cyclohexane is easier to penetrate the carbon bed at elevated temperature. So low temperature is beneficial to leakage test on the site, the recommend valve is not beyond $70^{\circ} \mathrm{C}$.

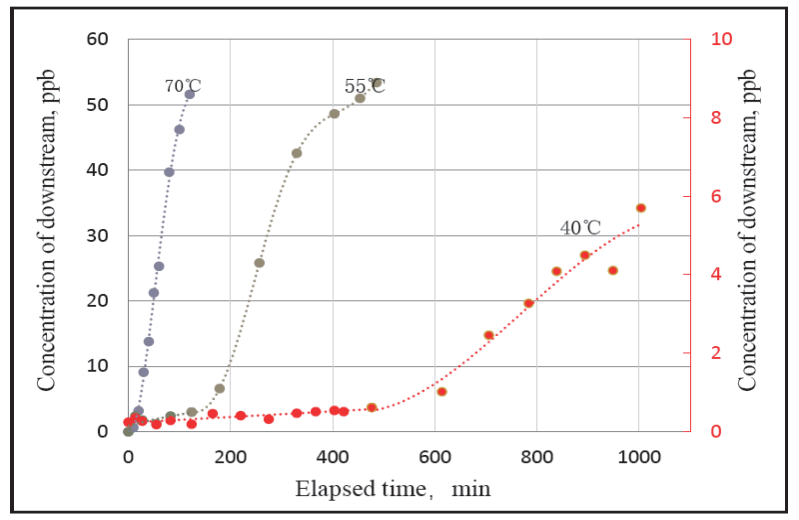

Figure 5. Desorption curve of cyclohexane with temperature. 


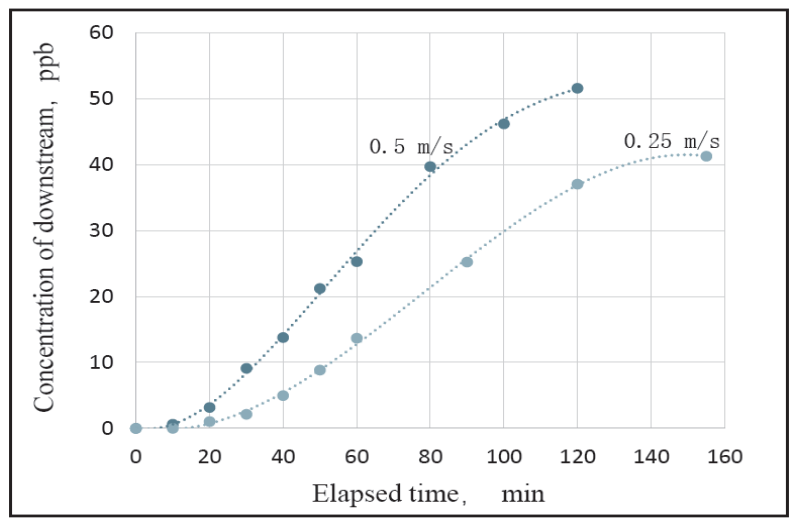

Figure 6. Desorption curve of cyclohexane with carrier gas flow rate.

\subsubsection{Flow rate}

The cyclohexane desorption curve (Figure 6) was obtain at a feed gas flow rate of $0.25 \mathrm{~m} / \mathrm{s}$ and $0.5 \mathrm{~m} / \mathrm{s}$. The experimental results show that the larger the carrier gas flow rate, the shorter the penetration time of cyclohexane on activated carbon, and the higher the concentration of cyclohexane in downstream of the activated carbon bed at the same time point. This is ascribed to the shorter

contact time of cyclohexane with activated carbon resulted by the higher flow rate of carrier gas, which is not conducive to the adsorption of cyclohexane. The larger carrier gas flow rate is also beneficial for desorption of the adsorbed cyclohexane from activated carbon. In order to avoid confusion between desorption and leakage, the onsite leakage test should be carried out on low air flow rate condition, not exceed $0.5 \mathrm{~m} / \mathrm{s}$.

\subsubsection{Upstream challenge gas concentration}

Figure 7 shows the effect of upstream tracer concentration on desorption time and desorption rate of cyclohexane under the same experimental conditions. The results show the penetration time of cyclohexane on activated carbon bed decreases with the increase of cyclohexane concentration in the upstream of activated carbon bed. This is due to the cyclohexane will occupy more active sites when the concentration increases. The performance is the adsorption mass transfer zone growth and the fresh area shorten. Therefore, the movement of cyclohexane in the activated carbon bed will be accelerated, and the penetration concentration will increase. So, In the field test, the injection of cyclohexane can not be too much, preferably the upstream concentration is not more than 200ppm.

\subsection{Leakage rate test}

\subsubsection{Leakage simulation test}

When the gas stream containing cyclohexane passes through the iodine adsorber, all of the organic components in the gas stream are almost retained by the activated carbon. When the carbon adsorbent itself collapses or is not installed properly, a portion of the gas stream does not pass through the activated carbon layer

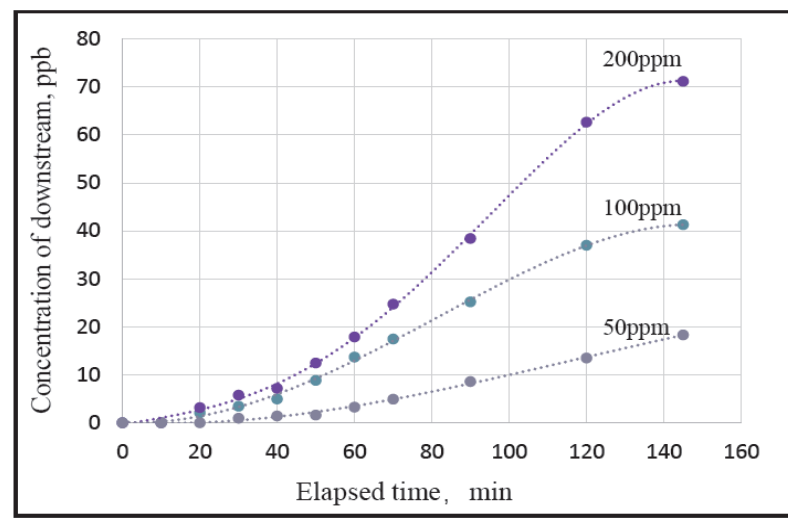

Figure7. Desorption curve of cyclohexane with gas concentration.

bypassing the iodine adsorber directly, resulting in an increase in the concentration of cyclohexane in the downstream gas stream. Therefore, by simulating leaks of different diameters, the effectiveness and sensitivity of this test method for small leaks can be verified. In this experiment, tiny bypass leak points of $0 \mathrm{~mm}, 1 \mathrm{~mm}, 2 \mathrm{~mm}$ and $3 \mathrm{~mm}$ in diameter were respectively created, and the leak rate was measured respectively by the cyclohexane test method.

Experimental results show that the well-structured iodine adsorber installed in the system, the leakage rate is $2.58 \times 10^{-5}$. When there is a $1 \mathrm{~mm}$ bypass hole, the leakage rate increases significantly to $2.67 \times 10^{-4}$, indicating that the test method can very sensitively detect tiny mechanical leaks. The assessment of the leakage rate depends on the size of the leakage point pore size. As can be seen from Figure 8, the leakage rate increases with the increase of bypass diameter, that means the cyclohexane method has a good response for mechanical leakage, and is feasible to evaluate the leakage rate for iodine adsorber.

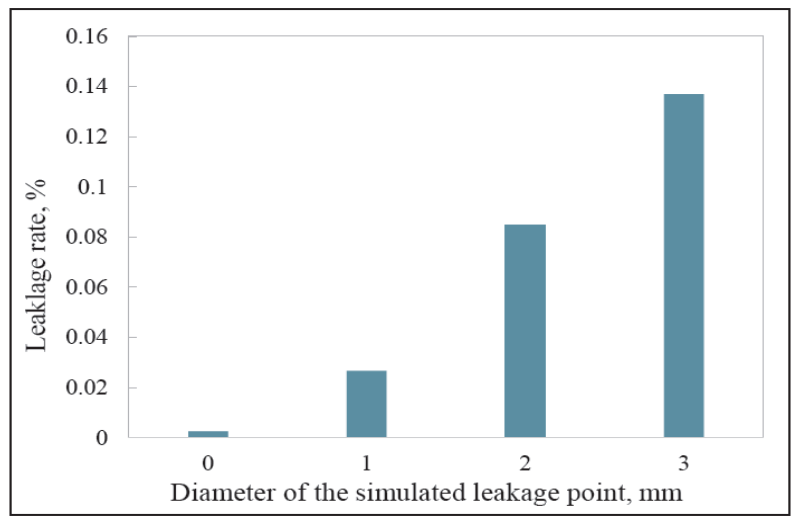

Figure 8. Leakage rate curve with different bypass diameters.

\subsubsection{Leakage rate comparison test}

Freon method uses the R-11 as a test reagent, measured with a halogen sensor. The principle of cyclohexane method is same as Freon method, but only the reagents and detection methods are different. The leakage rate was measured with freon method and 
cyclohexane method respectively under the conditions of different bypass diameters to verify whether these two test methods are consistent and comparable. In this experiment, bypass leaks of size $2 \mathrm{~mm} \times 3 \mathrm{~mm}, 2 \mathrm{~mm} \times$ $6 \mathrm{~mm}$ and $2 \mathrm{~mm} \times 10 \mathrm{~mm}$ were respectively created on iodine adsorber.

The result shows that when the iodine adsorber intact, the difference was only $7 \times 10^{-6}$ between the two methods measured results. When the sizes of leak holes were $2 \mathrm{~mm} \times 3 \mathrm{~mm}, 2 \mathrm{~mm} \times 6 \mathrm{~mm}$ and $2 \mathrm{~mm} \times 10 \mathrm{~mm}$, the difference between the two methods was only $3 \times 10^{-5}, 8 \times 10^{-5}$ and 7 $\times 10^{-5}$. Experimental results show that the leakage rates of the same iodine adsorber measured with freon method and cyclohexane method are almost the same value, that means cyclohexane method can be used in nuclear industry to replace the freon method to measure leakage rates of iodine adsorbers.

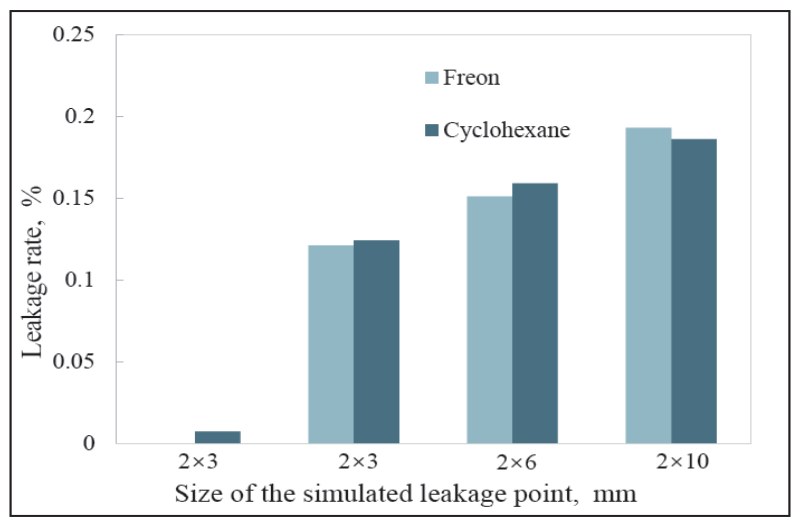

Figure 9. Leakage rates of an iodine adsorber for cyclohexane and freon.

\subsubsection{Precision verification}

A bypass leakage was created in the tested iodine adsorber and repeatability tests were conducted with cyclohexane method to verify dispersion degree of this method.

It can be known from the experimental results that the standard deviation of 6 repeated tests is $3.37 \times 10^{-5}$, the relative standard deviation is $2.71 \%$, and the confidence interval at $95 \%$ confidence level is $\left(1.17 \times 10^{-3}\right.$ to $\left.1.31 \times 10^{-3}\right)$. That is to say the cyclohexane method for leakage test of iodine adsorber has higher precision and reliability.

\section{Conclusion}

Our study on dynamic desorption behavior of cyclohexane gas on impregnated activated carbon shows that the retention time of cyclohexane at various conditions

\begin{tabular}{ccc} 
Table 1. & Repeatability-test results of cyclohexane method. \\
\hline Test No. & Leakage rate & Relative deviation \\
\hline 1 & $1.21 \times 10^{-3}$ & $-2.68 \%$ \\
2 & $1.20 \times 10^{-3}$ & $-3.48 \%$ \\
3 & $1.27 \times 10^{-3}$ & $+2.14 \%$ \\
4 & $1.28 \times 10^{-3}$ & $+2.95 \%$ \\
5 & $1.21 \times 10^{-3}$ & $-2.68 \%$ \\
6 & $1.29 \times 10^{-3}$ & $+3.75 \%$ \\
\hline
\end{tabular}

can meet the requirements for leakage test of iodine adsorbers. The new method is efficient to evaluate leakage rate of iodine adsorber. It also has advantages of simple operation, low toxicity and damage to people and environment, and good repeatability, and can be used in adsorber routine test and on-site test.

\section{Acknowledgements}

The authors Acknowledge support from Dayabay Nuclear Power Operations and Management Co. 1td under contract No. 3100067659, Hainan Nuclear Power Co. ltd under contract No. CNPHNN14TS2N0894/00. We are also grateful to Dangui Qiu, Jianrong Hou and Yuan Zhang for valuable contributions and discussions.

\section{References}

[1] The American Society of the Mechanical Engineers, ASME N510-2007, Testing of Nuclear Air-cleaning Systems, (2007).

[2] China National Nuclear Corporation, Nuclear air purification systems - Determination of the purification factor of iodine adsorbents Radioactive methyl iodide method: EJ1183-2005[S]. Beijing: Nuclear Industry Standardization Institute, (2005).

[3] Q. Liu, Field test methods for HEPA filters and iodine adsorbers in nuclear power plant air purification systems[J]. Radiation Protection 14(6), (1994).

[4] The Institute of Suzhou Thermal Research, Experimental apparatus for adsorption efficiency of iodine adsorber and its experimental method [P], china patent: CN105738142A, 2011-01.

[5] J. Zhang, Y. Li and H. Wang, Experimental study on lowly concentrated $\mathrm{CH}_{3} \mathrm{I}$ measurement for iodine absorber efficiency evaluation[J], Radiation Protection 35(3), (2015).

[6] X. Fan and H. Li, Experimental study on nondestructive performance of iodine adsorber[J]. Public Communication of Science \& Technology 23, (2011).

[7] Shanghai Nuclear Engineering Research and Design Institute, Nuclear field cleaning system on-site inspection: EJ791-93 [S]. Beijing: China Nuclear Industry Corporation, (1993).

[8] P. Gontier, Method for Controlling Leakage Rate of Active Carbon Filters:20080210879 [P], Maintenance Securite Installation Service, 2008-09-04.

[9] X. Wang, X. Zhang and Y. Zhang, Cyclohexane and other organic matter simultaneous determination of gas chromatography $[\mathrm{J}]$, Journal of Environment and Health 24(7) (2007).

[10]J.A. Dean (American), Analytical Chemistry Handbook [M]. Beijing: Science Press, (2003). 